\section{Difficulties in assessing carcinogenic activity}

SIR,-Extensive research projects to examine the validity of short-term tests for carcinogens are bcing carried out. The distinction between carcinogens and inactive compounds is often not clear. Consideration of the data on some of the pairs of active and inactive compounds illustrates this. The difficulties include the limitation of the number of animals that can bc used and frequently the dose of material which the animals can tolerate. In comparing pairs of related compounds the product assigned as inactive should have been tested at higher levels, say 10 times, than the related carcinogen.

The process or processes of carcinogencsis are complex and probably involve several stages. In addition to complete carcinogens and "activating enzyme systems", initiators, promoters, cocarcinogens, antioxidants and other factors are involved. The assessment of carcinogenic activity itself is difficult; in many cases it can only be stated that the available data are such that "no evaluation of the carcinogenicity of this compound can be made". Evell among the substances which are accepted as carcinogens there is a millionfold difference in potency as expressed in the dose of saccharin compared with aflatoxin needed to induce tumours. There are compounds such as phenobarbitone and DDT which are considered to be carcinogenic by some criteria but are not universally accepted as such. The proof of the negative, that a substance is not carcinogenic, is even more difficult and in many cases the tests on so called "noncarcinogens" are inadequate.

2-Naphthylamine is carcinogenic to dogs. monkeys, hamsters and mice in large doses but not carcinogenic to rats. Although 1-Naphthylamine appears to be noncarcinogenic in hamsters and possibly in dogs, it has induced tumours when given to newborn or adult mice.

$3: 4$-Benzopyrene is one of the most thoroughly investigated carcinogenic compounds and is a complete carcinogen. Pyrene is, however, a potent cocarcinogen (Hoffmann \& Wynder J. Air Pollut. Control Ass. 13, 322; 1963 and Van Duuren \& Goldschmidt J. Nat. Cancer Inst. 56, 1237; 1976) and in the complex process of cancer induction it is probable that cocarcinogens are important.

$\beta$-Propiolactone is carcinogenic on injection to rats and micc or skin application to mice. Although

$\gamma$ Butyrolactone was not active by oral administration or injection in micc (Rudali, Bovland \& Castegnaro C.R. Acad. Sci. 282, 799; 1976) it appeared to induce cancer in rats by oral administration (Schoental Israci J. Med. Sci. 4, 1146; 1968).

9, 10-Dimethylanthracene is a weak carcinogen on application to the skin, but inactive on injection in mice. Although anthracene was not carcinogenic when tested by skin application to mice, it induced sarcomas on injection in rats; (Schmähl Zeit. Krebsforsch 60, 697; 1955) naphthalenc was inactive when tested at the same time.

2-Acetylaminofluorene is one of the widely used experimental carcinogens that might have been used as an insecticide in food had tests not shown it to be carcinogenic. 4-Acetylaminofluorene has not been adequately investigated to say that it is inactive and in one experiment it induced cancer of the colon in one of ten rats.

Urethane is a complete carcinogen; although $O$-isopropyl-N-3-chluropheny] has not been shown to be a complete carcinogen, it acts as an initiator of tumours in mice skin when the original application is followed by treatment with croton oil.

With some types of compound chemical and biochemical considerations give indications of inactivity that may be more convincing than the available biological data. Thus the reactive 3 position of the reactive metabolite of 4-Nitroquinoline $N$ oxide is blocked in 3-methyl-4 nitroquinoline $N$ oxide, so that the latter is inactive. Similarly, in $3,3^{\prime}, 5,5^{\prime}$ tetramethylbenzidine the reactive ortho positions to the reactive hydroxylamine metabolite are blocked with methyl groups and the compound is inactive.

These examples show how difficult it is to be sure that compounds can be free of cancer hazard from animal tests. It is difficult to show that toxic compounds such as arsenic and selenium derivatives are carcinogenic to animals because only small doses can be given. On the other hand, substances with low acute toxicity such as saccharin and trichlorethylene have produced tumours because they can and were given in large amounts. The significance of the results of tests in which large amounts of otherwise harmless substances are administered is difficult to evaluate.

The uncertainty of negative results and the great quantitive variation in carcinogenic activity must be considered when short-term tests are compared with conventional tests for carcinogenic activity.

Yours faithfully,

ERIC BoYlani

The London School of Hyiene and Tropical Medicine, UK

\section{Was war the origin of intelligence?}

Sir.-- -In the review by Glynn Ll. Isaac on the development of early man (Nature 273,$588 ; 1978$ ) he suggests the question: "Was the enlargement of the brain in the Homo lineage(s) a response to the demands of culture? - or might a somewhat enlarged brain. at least at the outset, have been a preadaptation which facilitated the development of culture and technology?"
It is difficult to believe in preadaptations, and as I have suggested earlier (Fremlin. J. H. Be Fruitful and Multiply Rupert Hart-Davis 1972) the exceedingly rapid expansion of the brain is much more easily understood in terms of lethal competition between small closely-related groups. By the time our ancestors had developed the most elementary stone weapons they must have been very unattractive prey for the big carnivores and their subsequent population numbers must surely have been limited by direct competition between groups for food and the territory containing it. While a very moderate degree of intelligence is sufficient for the out-manocuvring of the big cats, there is no limit to the level of intelligence useful in competing with one's own species; whatever the current general level of intelligence, there is a great military advantage to any group which has a higher level still. Yours faithfully J. H. FREMLIN

University of Birmingham, UK

\section{Support for Spanish scientists}

SiR,_The article 'Spain has forgotten her scientists' (6 July, page 8), should undoubtedly bring the problems of Spanish science to the attention of important sectors of opinion in Spain and abroad.

We would like to emphasise the enthusiasm and dedication of a substantial number of Spanish scientists, who fecl capable of giving Spanish science a strong push forward if the minimal financial aid and basic facilities are provided. This is in contrast to the traditional apathy the Spanish have towards research, and their peculiar lack of confidence in their own scientists. The lack of science in the programmes of the political parties (mentioned in the article) is just a reflection of the lack of interest in the population as a whole. This, in turn, shows clcarly the poor educational standards of the country.

Industry entirely owned by Spanish capital is very rarc, and does not promote research; multinationals prefer to do their research in other, scientifically developed, countries. However, in both cases, this situation will change when the average quality of Spanish rescarch bccomes respectable. It is not vet appropriate to discuss the distinction between pure and applied science : applicd research will become possible only after a body of 'pure scientists' has been developed from which future 'applied' scientists will be trained. recycled. and with whom at all times. they will be able to interact.

$$
\text { Yours faithfully, }
$$

JuAN C. GOMEZ

Royal Free Hospital

FELIX M. GONI

School of Medicine.

London, UK 